\title{
Genetic susceptibility of opioid receptor genes polymorphism to drug addiction: A candidate-gene association study
}

\author{
Laith N. AL-Eitan ${ }^{1,2^{*}}$, Doaa M. Rababa'h and Mansour A. Alghamdi ${ }^{3,4}$
}

\begin{abstract}
Background: Like other complex diseases including drug addiction, genetic factors can interfere with the disease. In this study, three opioid genes (OPRM1, OPRD1, and OPRK1) were examined for an association with drug addiction among Jordanian males.

Methods: The study involved 498 addicts, in addition to 496 healthy controls and all from Arab descent.

Results: The findings in this study showed that rs1799971 of the OPRM1 gene was in association with drug addiction for both alleles and genotypes with $P$-values $=0.002$ and 0.01 , respectively. In addition, a significant association between the dominant model (A/A vs G/A-G/G) of rs1799971 (OPRM1) and drug addiction ( $P$-value = $0.003, \mathrm{OR}=1.59(1.17-2.15)$ ) was detected. Moreover, a genetic haplotype (AGGGCGACCCC) of theOPRM1 gene revealed a significant association with drug addiction ( $P$-value $=0.01, \mathrm{OR}=1.56(1.15-2.12)$ ). We also found that the age of addicts, smoking, and marital status with genetic variants within OPRM1, OPRD1, and OPRK1 genes may be implicated in drug addiction risk.
\end{abstract}

Conclusion: We propose that rs1799971 of the OPRM1gene is a genetic risk factor for drug addiction among Jordanian males.

Keywords: Opioids, Polymorphism, Drug addiction, Jordan

\section{Background}

Drug addictions are chronic complex diseases that featured by repetitive and irresistible consumption of certain drugs that lead to temporary euphoria. Subsequently, the addict will encounter tolerance, craving, and relapse as a result of neurological changes [1]. Up to $5 \%$ of the adults worldwide have been reported as drug abusers in (2010), drug addiction burden both society and individual's life in many aspects including physical, mental, and social aspects. Moreover, over 200

\footnotetext{
* Correspondence: Ineitan@just.edu.jo

'Department of Applied Biological Sciences, Jordan University of Science and Technology, Irbid 22110, Jordan

${ }^{2}$ Department of Biotechnology and Genetic Engineering, Jordan University of Science and Technology, Irbid 22110, Jordan

Full list of author information is available at the end of the article
}

thousand deaths annually are recording due to drug addiction [2]. Addiction has been linked to environmental factors together with the genetic one [3]. Inspecting the role of genetic variations in the etiology of the addiction may improve response to the treatments and help in disease prevention $[4,5]$. Both genome-wide methods and candidate gene studies, looking for the underlying role of the genetic factor in drug addiction by identification of genes that are crucial to neuroadaptation [6].

Opioid receptors (OPR) are part of the opioid system and responsible for mediating drug reward and addiction. These receptors in the nervous system attached to substances and induce a series of chemical reactions that lead to pain relief [7]. The OPRM1 gene located on chromosome number 6 and encode for endogenous $G$

C C The Author(s). 2020 Open Access This article is licensed under a Creative Commons Attribution 4.0 International License, which permits use, sharing, adaptation, distribution and reproduction in any medium or format, as long as you give appropriate credit to the original author(s) and the source, provide a link to the Creative Commons licence, and indicate if changes were made. The images or other third party material in this article are included in the article's Creative Commons licence, unless indicated otherwise in a credit line to the material. If material is not included in the article's Creative Commons licence and your intended use is not permitted by statutory regulation or exceeds the permitted use, you will need to obtain permission directly from the copyright holder. To view a copy of this licence, visit http://creativecommons.org/licenses/by/4.0/ The Creative Commons Public Domain Dedication waiver (http://creativecommons.org/publicdomain/zero/1.0/) applies to the data made available in this article, unless otherwise stated in a credit line to the data. 
protein-coupled mu-opioid receptors [8, 9]. The OPRM1 is one of the most studied genes regarding drug addiction and several genetic variants within the gene were significantly implicated with drug addiction. (rs1799971) SNP of the OPRM1 gene has been extensively investigated in association with drug addiction in different ethnic groups including Caucasian [10] European American, African American [11], German [12], and Japanese [13].

In addition, the OPRD1 and OPRK1 genes encode for the $\delta$-opioid receptor (DOR) and $\mathrm{K}$-opioid receptor (KOR) respectively are also belong to the opioid system. The OPRD1gene located on chromosome 1, while the OPRK1 gene located on chromosome 8 [14]. Activation of the $\mu$-opioid receptor and $\delta$-opioid receptor mediate drug rewarding DOR has been assigned as mood regulator for anxiety and depression [15]. Within the OPRD1 gene, both rs1042114 (G80T) and rs2234918 (T921C) have been in the spotlight for association with drug addiction. The latter SNP found to be correlated with heroin addiction in a German population [16]. Furthermore, the two intronic SNPs (rs2236857 and rs581111) within the OPRD1 gene found to be linked with heroin addiction risk [17].

Areas $\mathrm{k}$-opioid receptor activation is associated with increases in both physical and psychological stress responses. Genetic polymorphisms of the OPRK1 gene have been previously studied in association with substances [18]. In this study, we investigated the genetic susceptibility of three OPR genes (OPRM1, OPRD1 and OPRK1) to drug addiction among Jordanian males by screening and analyzing 29 candidate SNPs.

\section{Methods}

\section{Participants}

The study cohort consisted of 498 drug addicts selected depending on drug addiction criteria according to the Manual of Mental Disorders (DSM-IV) criteria [19]. All participants were males from Jordanian and were hospitalized in 2018 for 8 months in the National Centre for Rehabilitation of Addicts (NCRA) of the ministry of health in Jordanian and the Drug Rehabilitation Centre of the Jordanian Public Security Directorate (DRC-PSD). In addition, 496 healthy Jordanian male subjects with no history of drug addiction or psychiatric disorders were chosen as controls. Clinical data and structured questionnaires were collected according to the Human Ethics Committee of the Jordanian Ministry of Health $(\mathrm{MOH} /$ REC/180057), and the Institutional Review Board (IRB) at Jordan University of Science and Technology (43/114/ 2018). Patients who meet the inclusion criteria and had none of the exclusion criteria were interviewed, had the study objectives explained to them, and were asked to sign a consent form to participate in the study. Required written informed consent has been also obtained from all participants. All methods of the current study were performed in accordance with the IRB guidelines and regulations. Finally, all data was coded and no specific individual was identified.

\section{SNPs selection}

In this study, the 29 SNPs of three OPR genes (OPRM1, OPRD1, and OPRK1) were selected based on their clinical significance and according to early publications that reveled the plausible implication of these variants with drug addiction among other populations which make them polymorphisms of interest to be investigated as most of them have robust functions in neuroadaptation [20, 21]. Moreover, these polymorphisms selected to declare their influence on the Jordanian population of Arab descent.

\section{DNA extraction and SNP genotyping}

Venous blood samples were used to purify genomic DNA according to Wizard ${ }^{\circ}$ Genomic DNA Purification Kit (Promega Corporation, USA). Agarose gel electrophoresis and the Nano-Drop ND-1000 UV-Vis Spectrophotometer (BioDrop, UK) were used to select the quality and quantity of the extracted genetic material. Candidate SNPs within (OPRM1, OPRD1, and OPRK1) were screened using the Sequencing technique. Selected samples for genotyping were diluted using nuclease-free water with a final concentration of $20 \mathrm{ng} / \mu \mathrm{l}(50-500 \mu \mathrm{l})$ and shipped on wet ice to the Australian Genome Research Facility (AGRF) (Australia). At the AGRF, the samples were genotyped using the Agena Bioscience MassARRAY ${ }^{\circ}$ on a Compact Spectrometer, iPLEX GOLD chemistry.

\section{Statistical analysis}

The $P$-Value of Hardy-Weinberg equilibrium (HWE), was used to detect if the selected variants fulfill the (HWE) equation. Genotypic and allelic frequencies, genetic association, multiple genetic models, and haplotyping analyses were performed using SNPStats software (InstitutCatalàd'Oncologia, 2006). In addition, multinomial logistic regression was done using the Statistical Package for the Social Sciences (SPSS), version 25.0 (SPSS, Inc., Chicago, IL). P-values less than 0.05 were considered to be statistically significant.

\section{Multiple testing corrections}

The effective number of SNPs were tested according to [22] method, in addition, the Bonferroni correction was used to sets the significance cut-off at $\alpha / \mathrm{n}$ where $\alpha=$ 0.05 and $n$ number of tests [23]. 


\section{Results}

\section{Clinical characteristic of participants}

All participants in this study were Jordanian males of Arab descent. Four hundred ninety-eighr cases were identified as drug addicts with respect to different substances including; synthetic cannabinoids (47.5\%), cannabinoids (19.6\%), amphetamine (5.7\%), alcohol (5.5\%), benzodiazepines $(4.6 \%)$, opiates $(4.4 \%)$, cocaine $(1.1 \%)$, and cannabis (0.4\%). Among the cases $89 \%$ used only one substance. However, $11 \%$ of the addicts in this study were multi substance user. The routes for substances consumption were varying between substances. For instance, smoking was commonly used for cannabinoids use, while opiate was administrated by the cases using injection route. In addition, benzodiazepines, alcohol, and amphetamine were consumed orally. Cases were using inhalation for cocaine substance use.

The range of study cohort's ages was from 18 to 40 years with mean age $29 \pm 6.9$ for controls and $28.7 \pm 9.3$ for patients. Age, smoking, work, and marital status for cases were considered also in this study; $88.2 \%$ of the patients were smokers, (70.4\%) were single and (26.8\%) were unemployed.

\section{SNPs characterization}

Table 1 summarizes the list of selected genetic variants of three OPR genes (OPRM1, OPRD1, and OPRK1). The table also shows the chromosomal positions of SNPs, minor alleles and their frequencies, and the $P$-value of HWE for cases and controls. Among the investigated

Table 1 SNPs information and minor allele frequencies among cases and controls

\begin{tabular}{|c|c|c|c|c|c|c|c|c|}
\hline \multirow[t]{2}{*}{ Gene } & \multirow[t]{2}{*}{ SNP ID } & \multirow{2}{*}{$\begin{array}{l}\text { SNP } \\
\text { position }^{a}\end{array}$} & \multicolumn{3}{|c|}{ Cases $(n=498)$} & \multicolumn{3}{|c|}{ Controls $(n=496)$} \\
\hline & & & $\mathrm{MA}^{\mathrm{b}}$ & MAF $^{c}$ & HWE $^{d} P$-value & $\mathrm{MA}^{\mathrm{b}}$ & $\mathrm{MAF}^{\mathrm{c}}$ & HWE $^{\text {d } P \text {-value }}$ \\
\hline \multirow[t]{12}{*}{ OPRM1 } & rs648893 & $6: 154117494$ & $\mathrm{G}$ & 0.26 & 0.29 & G & 0.27 & 0.49 \\
\hline & rs609148 & $6: 154109880$ & $A$ & 0.26 & 0.56 & $A$ & 0.28 & 0.91 \\
\hline & rs495491 & $6: 154061407$ & G & 0.21 & 0.02 & G & 0.21 & 0.14 \\
\hline & rs3823010 & $6: 154058017$ & A & 0.08 & 1.00 & A & 0.09 & 0.58 \\
\hline & rs1799971 & $6: 154039662$ & G & 0.14 & 1.00 & G & 0.10 & 0.80 \\
\hline & rs511435 & $6: 154047412$ & $\mathrm{~T}$ & 0.16 & 0.5 & $\mathrm{~T}$ & 0.18 & 0.36 \\
\hline & rs524731 & $6: 154053957$ & $A$ & 0.15 & 0.39 & $A$ & 0.17 & 0.63 \\
\hline & rs1381376 & $6: 154072123$ & $\mathrm{~T}$ & 0.08 & 1.00 & $\mathrm{~T}$ & 0.1 & 0.30 \\
\hline & rs3778156 & $6: 154083178$ & $\mathrm{G}$ & 0.08 & 0.54 & G & 0.09 & 0.59 \\
\hline & rs2075572 & $6: 154090869$ & $G$ & 0.44 & 0.27 & G & 0.48 & 0.24 \\
\hline & rs548646 & $6: 154097012$ & $\mathrm{~T}$ & 0.32 & 0.41 & $\mathrm{~T}$ & 0.34 & 0.32 \\
\hline & rs671531 & $6: 154119607$ & $A$ & 0.32 & 0.68 & A & 0.34 & 0.16 \\
\hline \multirow[t]{6}{*}{ OPRK1 } & rs12675595 & $8: 53255365$ & $A$ & 0.1 & 0.79 & A & 0.1 & 0.44 \\
\hline & rs1051660 & $8: 53251002$ & $A$ & 0.11 & 0.64 & A & 0.12 & 0.53 \\
\hline & rs6985606 & $8: 53248556$ & $\mathrm{~T}$ & 0.33 & 1.00 & $\mathrm{~T}$ & 0.33 & 1.00 \\
\hline & rs997917 & 8:53239818 & C & 0.46 & 0.59 & C & 0.44 & 0.14 \\
\hline & rs702764 & 8:53229597 & C & 0.20 & 0.89 & C & 0.20 & 0.21 \\
\hline & rs963549 & 8:53229264 & $\mathrm{T}$ & 0.2 & 1.00 & $\mathrm{~T}$ & 0.2 & 0.21 \\
\hline \multirow[t]{10}{*}{ OPRD1 } & rs569356 & 1:28810174 & G & 0.08 & 0.75 & G & 0.07 & 1.00 \\
\hline & rs1042114 & $1: 28812463$ & $\mathrm{G}$ & 0.08 & 1.00 & G & 0.08 & 0.19 \\
\hline & rs678849 & 1:28818676 & $\mathrm{T}$ & 0.46 & 0.36 & $\mathrm{~T}$ & 0.45 & 0.86 \\
\hline & rs2236857 & 1:28835097 & C & 0.35 & 0.14 & C & 0.38 & 1.00 \\
\hline & rs2236855 & 1:28835487 & A & 0.34 & 0.13 & A & 0.38 & 1.00 \\
\hline & rs2298896 & 1:28839626 & $\mathrm{G}$ & 0.41 & 0.30 & G & 0.45 & 0.59 \\
\hline & rs421300 & 1:28843081 & G & 0.42 & 0.58 & G & 0.45 & 0.32 \\
\hline & rs529520 & $1: 28848434$ & C & 0.46 & 0.27 & C & 0.43 & 0.07 \\
\hline & rs12749204 & 1:28849701 & $G$ & 0.23 & 0.61 & G & 0.25 & 0.72 \\
\hline & rs2234918 & $1: 28863085$ & $\mathrm{~T}$ & 0.46 & 0.86 & $\mathrm{~T}$ & 0.46 & 0.79 \\
\hline
\end{tabular}

${ }^{\mathrm{a}}$ Chromosomal positions. ${ }^{\mathrm{b}}$ MA minor allele. ${ }^{\mathrm{c}}$ MAF minor allele frequency, ${ }^{\mathrm{d}}$ HWE Hardy-Weinberg equilibrium 
Table 2 Association of the investigated candidate gene polymorphisms with drug addiction

\begin{tabular}{|c|c|c|c|c|c|c|}
\hline \multirow[t]{2}{*}{ Gene } & \multirow[t]{2}{*}{ SNP ID } & \multicolumn{5}{|c|}{ Allelic and Genotypic Frequencies in Cases and Controls } \\
\hline & & Allele/Genotype & Cases $(n=498)$ & Controls $(n=496)$ & $P$-value* & Chi-square \\
\hline \multirow[t]{43}{*}{ OPRM1 } & rs648893 & A & $734(0.74)$ & $723(0.73)$ & 0.57 & 0.31 \\
\hline & & G & $256(0.26)$ & $267(0.27)$ & & \\
\hline & & AA & $267(0.54)$ & $267(0.54)$ & 0.34 & 2.1 \\
\hline & & AG & $200(0.4)$ & $189(0.38)$ & & \\
\hline & & GG & $28(0.06)$ & $39(0.08)$ & & \\
\hline & rs609148 & G & $729(0.74)$ & $709(0.72)$ & 0.24 & 1.3 \\
\hline & & A & $255(0.26)$ & $279(0.28)$ & & \\
\hline & & GG & $267(0.54)$ & $255(0.52)$ & 0.42 & 1.7 \\
\hline & & GA & $195(0.4)$ & $199(0.4)$ & & \\
\hline & & AA & $30(0.06)$ & $40(0.08)$ & & \\
\hline & rs3823010 & G & $905(0.92)$ & $901(0.91)$ & 0.44 & 0.58 \\
\hline & & A & 79 (0.08) & 89 (0.09) & & \\
\hline & & GG & $416(0.85)$ & $411(0.83)$ & 0.68 & 0.75 \\
\hline & & GA & $73(0.15)$ & $79(0.16)$ & & \\
\hline & & $A A$ & $3(0.01)$ & $5(0.01)$ & & \\
\hline & rs1799971 & A & $848(0.86)$ & $894(0.9)$ & 0.002 & 9.4 \\
\hline & & G & $140(0.14)$ & $96(0.1)$ & & \\
\hline & & AA & $364(0.74)$ & $404(0.82)$ & 0.01 & 9.3 \\
\hline & & GA & $120(0.24)$ & $86(0.17)$ & & \\
\hline & & GG & $10(0.02)$ & $5(0.01)$ & & \\
\hline & rs511435 & C & $833(0.84)$ & $811(0.82)$ & 0.18 & 1.7 \\
\hline & & T & $155(0.16)$ & $177(0.18)$ & & \\
\hline & & $C C$ & $353(0.71)$ & $336(0.68)$ & 0.42 & 1.7 \\
\hline & & $\mathrm{CT}$ & $127(0.26)$ & $139(0.28)$ & & \\
\hline & & $\Pi$ & $14(0.03)$ & $19(0.04)$ & & \\
\hline & rs524731 & $C$ & $835(0.85)$ & $824(0.83)$ & 0.43 & 0.60 \\
\hline & & A & $151(0.15)$ & $164(0.17)$ & & \\
\hline & & $\mathrm{CC}$ & $356(0.72)$ & $345(0.7)$ & 0.71 & 0.67 \\
\hline & & $C A$ & $123(0.25)$ & $134(0.27)$ & & \\
\hline & & $A A$ & $14(0.03)$ & $15(0.03)$ & & \\
\hline & rs1381376 & $C$ & $907(0.92)$ & $893(0.9)$ & 0.12 & 2.4 \\
\hline & & T & 77 (0.08) & $97(0.1)$ & & \\
\hline & & CC & $418(0.85)$ & $405(0.82)$ & 0.25 & 2.7 \\
\hline & & $C T$ & $71(0.14)$ & $83(0.17)$ & & \\
\hline & & $\pi$ & $3(0.01)$ & $7(0.01)$ & & \\
\hline & rs3778156 & A & 909 (0.92) & $899(0.91)$ & 0.34 & 0.9 \\
\hline & & G & $79(0.08)$ & $91(0.09)$ & & \\
\hline & & $A A$ & $419(0.85)$ & $409(0.83)$ & 0.64 & 0.88 \\
\hline & & $A G$ & $71(0.14)$ & $81(0.16)$ & & \\
\hline & & GG & $4(0.01)$ & $5(0.01)$ & & \\
\hline & rs2075572 & $C$ & $550(0.56)$ & $518(0.52)$ & 0.11 & 2.5 \\
\hline & & G & $434(0.44)$ & $472(0.48)$ & & \\
\hline & & CC & $160(0.33)$ & $142(0.29)$ & 0.30 & 2.4 \\
\hline
\end{tabular}


Table 2 Association of the investigated candidate gene polymorphisms with drug addiction (Continued)

\begin{tabular}{|c|c|c|c|c|c|c|}
\hline \multirow[t]{2}{*}{ Gene } & \multirow[t]{2}{*}{ SNP ID } & \multicolumn{5}{|c|}{ Allelic and Genotypic Frequencies in Cases and Controls } \\
\hline & & Allele/Genotype & Cases $(n=498)$ & Controls $(n=496)$ & $P$-value* & Chi-square \\
\hline & & CG & $230(0.47)$ & $234(0.47)$ & & \\
\hline & & GG & $102(0.21)$ & $119(0.24)$ & & \\
\hline & rs548646 & C & $648(0.68)$ & $640(0.66)$ & 0.33 & 0.93 \\
\hline & & T & $310(0.32)$ & $336(0.34)$ & & \\
\hline & & $\mathrm{CC}$ & $223(0.47)$ & $215(0.44)$ & 0.63 & 0.91 \\
\hline & & $\mathrm{CT}$ & $202(0.42)$ & $210(0.43)$ & & \\
\hline & & $\pi$ & $54(0.11)$ & $63(0.13)$ & & \\
\hline & rs671531 & G & $669(0.68)$ & $653(0.66)$ & 0.33 & 0.92 \\
\hline & & A & $313(0.32)$ & $335(0.34)$ & & \\
\hline & & GG & $230(0.47)$ & $223(0.45)$ & 0.51 & 1.35 \\
\hline & & $\mathrm{GA}$ & $209(0.43)$ & $207(0.42)$ & & \\
\hline & & $A A$ & $52(0.11)$ & $64(0.13)$ & & \\
\hline \multirow[t]{30}{*}{ OPRK1 } & rs12675595 & G & $897(0.9)$ & $894(0.9)$ & 0.93 & 0.01 \\
\hline & & A & $95(0.1)$ & $96(0.1)$ & & \\
\hline & & GG & $406(0.82)$ & $405(0.82)$ & 0.95 & 0.1 \\
\hline & & GA & $85(0.17)$ & $84(0.17)$ & & \\
\hline & & AA & $5(0.01)$ & $6(0.01)$ & & \\
\hline & rs1051660 & $C$ & $882(0.897)$ & $869(0.88)$ & 0.36 & 0.83 \\
\hline & & A & $108(0.11)$ & $121(0.12)$ & & \\
\hline & & CC & $383(0.77)$ & $394(0.8)$ & 0.66 & 0.81 \\
\hline & & $C A$ & $103(0.21)$ & 94 (0.19) & & \\
\hline & & $A A$ & $9(0.02)$ & $7(0.01)$ & & \\
\hline & rs6985606 & C & $217(0.67)$ & $664(0.67)$ & NA & NA \\
\hline & & T & $327(0.33)$ & $324(0.33)$ & & \\
\hline & & $C C$ & $217(0.44)$ & $223(0.45)$ & 0.96 & 0.07 \\
\hline & & $\mathrm{CT}$ & $219(0.45)$ & $218(0.44)$ & & \\
\hline & & $\pi$ & $54(0.11)$ & $53(0.11)$ & & \\
\hline & rs997917 & T & $529(0.54)$ & $552(0.56)$ & 0.30 & 1.08 \\
\hline & & $C$ & $455(0.46)$ & $432(0.44)$ & & \\
\hline & & $\pi$ & $139(0.28)$ & $163(0.33)$ & 0.20 & 3.2 \\
\hline & & $\mathrm{TC}$ & $251(0.51)$ & $226(0.46)$ & & \\
\hline & & CC & $102(0.21)$ & $103(0.21)$ & & \\
\hline & rs702764 & T & $788(0.8)$ & $790(0.8)$ & 0.91 & 0.01 \\
\hline & & $C$ & $202(0.2)$ & $200(0.2)$ & & \\
\hline & & $\pi$ & $314(0.63)$ & $320(0.65)$ & 0.69 & 0.72 \\
\hline & & $\mathrm{TC}$ & $160(0.32)$ & $150(0.3)$ & & \\
\hline & & CC & $21(0.04)$ & $25(0.05)$ & & \\
\hline & rs963549 & C & $789(0.8)$ & $783(0.8)$ & 0.73 & 0.11 \\
\hline & & T & $195(0.2)$ & $201(0.2)$ & & \\
\hline & & $\mathrm{CC}$ & $316(0.64)$ & $316(0.64)$ & 0.62 & 0.93 \\
\hline & & $\mathrm{CT}$ & $157(0.32)$ & $151(0.31)$ & & \\
\hline & & $\pi$ & $19(0.04)$ & $25(0.05)$ & & \\
\hline OPRD1 & rs569356 & $A$ & $910(0.92)$ & $912(0.93)$ & 0.73 & 0.11 \\
\hline
\end{tabular}


Table 2 Association of the investigated candidate gene polymorphisms with drug addiction (Continued)

\begin{tabular}{|c|c|c|c|c|c|c|}
\hline \multirow[t]{2}{*}{ Gene } & \multirow[t]{2}{*}{ SNP ID } & \multicolumn{5}{|c|}{ Allelic and Genotypic Frequencies in Cases and Controls } \\
\hline & & Allele/Genotype & Cases $(n=498)$ & Controls $(n=496)$ & $P$-value* & Chi-square \\
\hline & & G & $74(0.08)$ & $70(0.07)$ & & \\
\hline & & AA & $421(0.86)$ & $423(0.86)$ & 0.89 & 0.23 \\
\hline & & $A G$ & $68(0.14)$ & $66(0.13)$ & & \\
\hline & & GG & $3(0.01)$ & $2(0.01)$ & & \\
\hline & rs1042114 & $\mathrm{T}$ & $912(0.92)$ & $912(0.92)$ & NA & NA \\
\hline & & G & $76(0.08)$ & $76(0.08)$ & & \\
\hline & & $\pi$ & $421(0.85)$ & $423(0.86)$ & 0.73 & 0.62 \\
\hline & & TG & $70(0.14)$ & $66(0.13)$ & & \\
\hline & & GG & $3(0.01)$ & $5(0.01)$ & & \\
\hline & rs678849 & C & $527(0.54)$ & $545(0.55)$ & 0.47 & 0.51 \\
\hline & & $\mathrm{T}$ & $455(0.46)$ & $441(0.45)$ & & \\
\hline & & CC & $136(0.28)$ & $149(0.3)$ & 0.68 & 0.76 \\
\hline & & $C T$ & $255(0.52)$ & $247(0.5)$ & & \\
\hline & & $\pi$ & $100(0.2)$ & $97(0.2)$ & & \\
\hline & rs2236857 & $\mathrm{T}$ & $644(0.65)$ & $610(0.62)$ & 0.10 & 2.7 \\
\hline & & C & $342(0.35)$ & $378(0.38)$ & & \\
\hline & & $\pi$ & $218(0.44)$ & $188(0.38)$ & 0.14 & 3.9 \\
\hline & & $\mathrm{TC}$ & $208(0.42)$ & $234(0.47)$ & & \\
\hline & & $\mathrm{CC}$ & $67(0.14)$ & $72(0.15)$ & & \\
\hline & rs2236855 & C & $649(0.66)$ & $612(0.62)$ & 0.06 & 3.6 \\
\hline & & A & $333(0.34)$ & $376(0.38)$ & & \\
\hline & & $\mathrm{CC}$ & $222(0.45)$ & 189 (0.38) & 0.08 & 4.9 \\
\hline & & CA & $205(0.42)$ & $234(0.47)$ & & \\
\hline & & $\mathrm{AA}$ & $64(0.13)$ & $71(0.14)$ & & \\
\hline & rs2298896 & $\mathrm{T}$ & $573(0.59)$ & $539(0.55)$ & 0.05 & 3.6 \\
\hline & & G & $401(0.41)$ & $449(0.45)$ & & \\
\hline & & $\pi$ & $174(0.36)$ & $150(0.3)$ & 0.16 & 3.6 \\
\hline & & $\mathrm{TG}$ & $225(0.46)$ & $239(0.48)$ & & \\
\hline & & GG & $88(0.18)$ & $105(0.21)$ & & \\
\hline & rs421300 & A & $559(0.58)$ & $540(0.55)$ & 0.18 & 1.8 \\
\hline & & G & $407(0.42)$ & $444(0.45)$ & & \\
\hline & & $\mathrm{AA}$ & $165(0.34)$ & $154(0.31)$ & 0.40 & 1.8 \\
\hline & & $A G$ & $229(0.47)$ & $232(0.47)$ & & \\
\hline & & GG & $89(0.18)$ & $106(0.22)$ & & \\
\hline & rs529520 & A & $523(0.54)$ & $556(0.57)$ & 0.19 & 1.7 \\
\hline & & C & $447(0.46)$ & $422(0.43)$ & & \\
\hline & & $\mathrm{AA}$ & $147(0.3)$ & $168(0.34)$ & 0.39 & 1.8 \\
\hline & & $A C$ & $229(0.47)$ & $220(0.45)$ & & \\
\hline & & CC & $109(0.22)$ & $101(0.21)$ & & \\
\hline & rs12749204 & A & $762(0.77)$ & $745(0.75)$ & 0.33 & 0.95 \\
\hline & & G & $226(0.23)$ & $245(0.25)$ & & \\
\hline & & $\mathrm{AA}$ & $296(0.6)$ & $282(0.57)$ & 0.62 & 0.94 \\
\hline & & $A G$ & $170(0.34)$ & $181(0.37)$ & & \\
\hline
\end{tabular}


Table 2 Association of the investigated candidate gene polymorphisms with drug addiction (Continued)

\begin{tabular}{|c|c|c|c|c|c|c|}
\hline \multirow[t]{2}{*}{ Gene } & \multirow[t]{2}{*}{ SNP ID } & \multicolumn{5}{|c|}{ Allelic and Genotypic Frequencies in Cases and Controls } \\
\hline & & Allele/Genotype & Cases $(n=498)$ & Controls $(n=496)$ & $P$-value* & Chi-square \\
\hline & & GG & $28(0.06)$ & $32(0.06)$ & & \\
\hline & \multirow[t]{5}{*}{ rs2234918 } & C & $536(0.54)$ & $536(0.54)$ & 0.96 & 0.002 \\
\hline & & $\mathrm{T}$ & $454(0.46)$ & $452(0.46)$ & & \\
\hline & & $\mathrm{CC}$ & $146(0.29)$ & $147(0.3)$ & 0.99 & 0.01 \\
\hline & & $\mathrm{CT}$ & $244(0.49)$ & $242(0.49)$ & & \\
\hline & & $\pi$ & $105(0.21)$ & $105(0.21)$ & & \\
\hline
\end{tabular}

${ }^{*} P$-Value $<0.05$ is considered as significant

NA Not Applicable

SNPs, rs495491 SNP of the OPRM1 gene did not fulfill the HWE eq. $(P$-value $<0.05)$ and were excluded from this study.

\section{Genetic association analyses}

The correlation between the studied SNPs and drug addiction was conducted by performing different statistical tests. Table 2 illustrates the differences in the allelic and genotypic distribution between patients and controls. Our findings exposed that only rs1799971 of the OPRM1 gene was in association with drug addiction in this study ( $P$-values were; $0.002,0.01)$ for alleles and genotypes difference respectively. A significant variation in the variant allele (G) frequency distribution was observed between cases and controls; $14 \%$ of patients carried the $(G)$ allele compared to only $1 \%$ of healthy participants with the same variant (Table 2). In addition, the genotype (GG) among patients was twice it among control, this finding may propose the $(\mathrm{G})$ allele of $\operatorname{rs} 1799971$ (OPRM1) as a risk genetic locus for drug addiction. Moreover, different genetic models analysis was done for further confirmation. Table S1 displays the included models in this study (dominant and recessive). According to Bonferroni correction, the $P$-value was set to 0.02 . The result show a significant association between the dominant model (A/A vs G/A-G/G) of rs1799971 (OPRM1) and drug addiction $(P$-value $=0.003$, OR $=1.59(1.17-2.15))$. Haplotyping was also investigated as a part of the genetic association's analyses in this study. As Table S2 demonstrates, one block of the OPRM1 gene (AGGGCGAC CCC) exhibits significant association with drug addiction $(P$-value $=0.01, \mathrm{OR}=1.56(1.15-2.12))$.

\section{Regression analysis}

In this study, regression analysis was done to detect the relationship between four major features that were significant for drug addiction among cases and the selected SNPs. Tables 3, 4, and 5 depict the outcome of regression analysis. However, age of the addicts, smoking and marital status revealed a relationship with different genotypes of some candidate SNPs of the investigated genes was only significant for the risk of drug addiction among males Jordanians.

\section{Discussion}

Drug addiction is a chronic disease that involves both the environmental and genetic factors [24]. Despite the vague knowledge about the robust role of the genetic parameters in drug addiction risk, several approaches were extensively investigated to put the baseline of genetic predisposition to drug addiction such as family, twin, and adoption studies [25-27]. This study focus on investigating the impact of many Single nucleotide variants within critical genes on drug addiction among Jordanians. Several previous studies conducted on Jordanian Arabs have investigated the involvement of candidate genes in drug addiction including $D R D 4,5$-HTTLPR [28] SLC6A4 [29] and OPRM1 [3, 30, 31].

Our findings revealed that the OPRM1 gene may be implicated in drug addiction among Jordanians, in particular the functioning SNP (rs1799971) found to be significantly associated with drug addiction which is in consistence with a Chinese study on336 Han heroin addicts [32]. In this study, the distribution of the variant genotype GG of rs1799971 within cases was twice it within controls which propose that the $G$ allele of (rs1799971) within OPRM1 gene may influence drug addiction increased risk. The aforementioned SNP is a missense variant located on exon 1 and encodes for a change from asparagine to aspartate amino acids. It has been suggested that this change results in removing an $\mathrm{N}$-glycosylation site in the extracellular domain which alters the activity of endogenous opioid binding receptors [33]. These actions end in pain harbor and reduction in the response to analgesic drugs [9, 32] furthermore, it tend to increase administration of opioids [31]. In contrast to this study finding [11, 13] reported no evidence of an association between rs1799971 polymorphism and opioid addiction among AfricanAmericans/ European-Americans (EA) and Japanese population. 
Table 3 Regression analysis of the association between drug addiction features and candidate SNPs of OPRM1 gene

\begin{tabular}{|c|c|c|c|c|}
\hline \multirow{2}{*}{$\frac{\text { SNP ID }}{\text { rs648893 }}$} & \multirow{2}{*}{$\begin{array}{l}\text { Covariate } \\
\text { Age }\end{array}$} & \multicolumn{2}{|c|}{ Regression coefficients B B vs Expected. B* } & \multirow{2}{*}{$\frac{P \text {-value }}{<0}$} \\
\hline & & 0.42 & 1.53 & \\
\hline & Smoking & -1.27 & 0.28 & $<0.0001$ \\
\hline & Work & 0.10 & 1.10 & 0.55 \\
\hline & Marital status & -1.34 & 0.26 & $<0.0001$ \\
\hline & GG & -0.28 & 0.75 & 0.33 \\
\hline & $A G$ & 0.11 & 1.11 & 0.44 \\
\hline & AA & reference & & \\
\hline \multirow[t]{7}{*}{ rs609148 } & Age & 0.42 & 1.52 & $<0.0001$ \\
\hline & Smoking & -1.27 & 0.28 & $<0.0001$ \\
\hline & Work & 0.08 & 1.09 & 0.61 \\
\hline & Marital status & -1.33 & 0.26 & $<0.0001$ \\
\hline & GG & 0.30 & 1.35 & 0.27 \\
\hline & AG & 0.29 & 1.33 & 0.31 \\
\hline & AA & reference & & \\
\hline \multirow[t]{7}{*}{ rs3823010 } & Age & 0.42 & 1.52 & $<0.0001$ \\
\hline & Smoking & -1.28 & 0.27 & $<0.0001$ \\
\hline & Work & 0.10 & 1.11 & 0.53 \\
\hline & Marital status & -1.34 & 0.26 & $<0.0001$ \\
\hline & GG & 0.07 & 1.07 & 0.92 \\
\hline & $A G$ & 0.06 & 1.06 & 0.93 \\
\hline & AA & reference & & \\
\hline \multirow[t]{7}{*}{ rs1799971 } & Age & 0.42 & 1.52 & $<0.0001$ \\
\hline & Smoking & -1.28 & 0.27 & $<0.0001$ \\
\hline & Work & 0.11 & 1.12 & 0.50 \\
\hline & Marital status & -1.34 & 0.26 & $<0.0001$ \\
\hline & GG & 0.73 & 2.08 & 0.23 \\
\hline & $A G$ & 0.47 & 1.61 & 0.01 \\
\hline & AA & reference & & \\
\hline \multirow[t]{7}{*}{ rs524731 } & Age & 0.42 & 1.53 & $<0.0001$ \\
\hline & Smoking & -1.30 & 0.27 & $<0.0001$ \\
\hline & Work & 0.06 & 1.07 & 0.62 \\
\hline & Marital status & -1.35 & 0.25 & $<0.0001$ \\
\hline & $\mathrm{CC}$ & -0.04 & 0.95 & 0.90 \\
\hline & $C A$ & -0.06 & 0.93 & 0.87 \\
\hline & AA & reference & & \\
\hline \multirow[t]{7}{*}{ rs511435 } & Age & 0.42 & 1.52 & $<0.0001$ \\
\hline & Smoking & -1.30 & 0.27 & $<0.0001$ \\
\hline & Work & 0.08 & 1.08 & 0.69 \\
\hline & Marital status & -1.33 & 0.26 & $<0.0001$ \\
\hline & $\mathrm{CC}$ & 0.18 & 1.20 & 0.63 \\
\hline & $\mathrm{CT}$ & 0.15 & 1.16 & 0.70 \\
\hline & $\pi$ & reference & & \\
\hline \multirow[t]{2}{*}{ rs1381376 } & Age & 0.41 & 1.51 & $<0.0001$ \\
\hline & Smoking & -1.27 & 0.28 & $<0.0001$ \\
\hline
\end{tabular}


Table 3 Regression analysis of the association between drug addiction features and candidate SNPs of OPRM1 gene (Continued)

\begin{tabular}{|c|c|c|c|c|}
\hline \multirow[t]{2}{*}{ SNP ID } & \multirow{2}{*}{$\begin{array}{l}\text { Covariate } \\
\text { Work }\end{array}$} & \multicolumn{2}{|c|}{ Regression coefficients B B vs Expected. B* } & \multirow{2}{*}{$\frac{P \text {-value }}{0.53}$} \\
\hline & & 0.11 & 1.11 & \\
\hline & Marital status & -1.32 & 0.26 & $<0.0001$ \\
\hline & CC & 0.33 & 1.39 & 0.65 \\
\hline & CT & 0.16 & 1.18 & 0.82 \\
\hline & $\pi$ & reference & & \\
\hline \multirow[t]{7}{*}{ rs3778156 } & Age & 0.44 & 1.55 & $<0.0001$ \\
\hline & Smoking & -1.26 & 0.28 & $<0.0001$ \\
\hline & Work & 0.10 & 1.10 & 0.56 \\
\hline & Marital status & -1.37 & 0.25 & $<0.0001$ \\
\hline & GG & 0.03 & 1.03 & 0.96 \\
\hline & AG & -0.19 & 0.82 & 0.31 \\
\hline & AA & reference & & \\
\hline \multirow[t]{7}{*}{ rs2075572 } & Age & 0.42 & 1.524 & $<0.0001$ \\
\hline & Smoking & -1.26 & 0.283 & $<0.0001$ \\
\hline & Work & 0.10 & 1.112 & 0.53 \\
\hline & Marital status & -1.34 & 0.262 & $<0.0001$ \\
\hline & $\mathrm{CC}$ & -0.18 & 0.833 & 0.34 \\
\hline & CG & -0.11 & 0.896 & 0.50 \\
\hline & GG & reference & & \\
\hline \multirow[t]{7}{*}{0063 rs548646 } & Age & 0.43 & 1.54 & $<0.0001$ \\
\hline & Smoking & -1.29 & 0.27 & $<0.0001$ \\
\hline & Work & 0.10 & 1.11 & 0.54 \\
\hline & Marital status & -1.31 & 0.26 & $<0.0001$ \\
\hline & CC & 0.05 & 1.05 & 0.81 \\
\hline & CT & 0.01 & 1.01 & 0.96 \\
\hline & $\pi$ & reference & & \\
\hline \multirow[t]{7}{*}{ rs671531 } & Age & 0.42 & 1.52 & $<0.0001$ \\
\hline & Smoking & -1.25 & 0.28 & $<0.0001$ \\
\hline & Work & 0.09 & 1.09 & 0.58 \\
\hline & Marital status & -1.34 & 0.26 & $<0.0001$ \\
\hline & GG & 0.15 & 1.16 & 0.49 \\
\hline & $A G$ & 0.14 & 1.15 & 0.52 \\
\hline & AA & reference & & \\
\hline
\end{tabular}

* Beta coefficients

** P-Value $<0.05$ is considered as significant

rs648893 (C/T) polymorphism an Intronic SNP of the OPRM1 gene that implicated in DNA splicing has been recently nominated as candidate for substance dependence including drugs [34]. In accordance to our study, $[34,35]$ found that the rs648893 of OPRM1 was not associated with drug addiction among Iranian population and European Americans respectively. After all, ethnic variations reflect the differences in the drug addiction etiology among populations. Another significant outcome of this recent study, we found that one block of the OPRM1 gene (AGGGCGACCCC) exhibits significant association with drug addiction among Jordanian males.

In this study, none of the investigated SNPs of both $O P R D 1$ and OPRK1 genes were in correlation with drug addiction among Jordanians. However, in a review of several studies about genetic variants of addiction susceptibility summarizes that both rs1051660 and rs1051660 of OPRK1 are involved in drug addiction [7], while rs1042114 polymorphism of OPRD1 was significantly correlated with substance dependence disorder among EA [19]. 
Table 4 Regression analysis of the association between drug addiction features and candidate SNPs of OPRK1 gene

\begin{tabular}{|c|c|c|c|c|}
\hline \multirow{2}{*}{$\frac{\text { SNP ID }}{\text { rs12675595 }}$} & \multirow{2}{*}{$\begin{array}{l}\text { Covariate } \\
\text { Age }\end{array}$} & \multicolumn{2}{|c|}{ Regression coefficients B B vs Expected. B* } & \multirow{2}{*}{$\frac{P \text {-value }}{0 . *}$} \\
\hline & & 0.30 & 1.35 & \\
\hline & Smoking & -1.25 & 0.28 & $<0.0001$ \\
\hline & Work & 0.18 & 1.19 & 0.28 \\
\hline & Marital status & -0.98 & 0.37 & $<0.0001$ \\
\hline & GG & 0.41 & 1.51 & 0.53 \\
\hline & $A G$ & 0.36 & 1.44 & 0.59 \\
\hline & AA & reference & & \\
\hline \multirow[t]{7}{*}{ rs1051660 } & Age & -0.30 & .735 & 0.01 \\
\hline & Smoking & 1.25 & 3.515 & $<0.0001$ \\
\hline & Work & -0.18 & .828 & 0.26 \\
\hline & Marital status & 0.97 & 2.656 & $<0.0001$ \\
\hline & $\mathrm{CC}$ & 0.20 & 1.223 & 0.730 \\
\hline & CA & 0.32 & 1.390 & 0.581 \\
\hline & $A A$ & reference & & \\
\hline \multirow[t]{7}{*}{ rs6985606 } & Age & -0.30 & 0.73 & 0.01 \\
\hline & Smoking & 1.24 & 3.46 & $<0.0001$ \\
\hline & Work & -0.16 & 0.84 & 0.32 \\
\hline & Marital status & 0.96 & 2.62 & $<0.0001$ \\
\hline & $\mathrm{CC}$ & 0.03 & 1.03 & 0.86 \\
\hline & $\mathrm{CT}$ & -0.01 & 0.98 & 0.96 \\
\hline & $\pi$ & reference & & \\
\hline \multirow[t]{7}{*}{ rs997917 } & Age & -0.29 & 0.74 & 0.01 \\
\hline & Smoking & 1.26 & 3.55 & $<0.0001$ \\
\hline & Work & -0.17 & 0.84 & 0.30 \\
\hline & Marital status & 0.94 & 2.56 & $<0.0001$ \\
\hline & $\mathrm{CC}$ & -0.20 & 0.81 & 0.28 \\
\hline & $\mathrm{CT}$ & -0.25 & 0.77 & 0.10 \\
\hline & $\pi$ & reference & & \\
\hline \multirow[t]{7}{*}{ rs702764 } & Age & -0.31 & 0.73 & 0.01 \\
\hline & Smoking & 1.26 & 3.55 & $<0.0001$ \\
\hline & Work & -0.17 & 0.84 & 0.30 \\
\hline & Marital status & 0.97 & 2.64 & $<0.0001$ \\
\hline & $\mathrm{CC}$ & -0.09 & 0.91 & 0.78 \\
\hline & $\mathrm{CT}$ & -0.15 & 0.86 & 0.31 \\
\hline & $\Pi$ & reference & & \\
\hline \multirow[t]{7}{*}{ rs963549 } & Age & -0.30 & 0.73 & 0.01 \\
\hline & Smoking & 1.26 & 3.54 & $<0.0001$ \\
\hline & Work & -0.18 & 0.83 & 0.28 \\
\hline & Marital status & 0.96 & 2.61 & $<0.0001$ \\
\hline & $\mathrm{CC}$ & 0.01 & 1.01 & 0.97 \\
\hline & $C T$ & -0.09 & 0.90 & 0.78 \\
\hline & $\pi$ & reference & & \\
\hline
\end{tabular}


Table 5 Regression analysis of the association between drug addiction features and candidate SNPs of OPRD1 gene

\begin{tabular}{|c|c|c|c|c|}
\hline \multirow{2}{*}{$\frac{\text { SNP ID }}{\text { rs569356 }}$} & \multirow{2}{*}{$\begin{array}{l}\text { Covariate } \\
\text { Age }\end{array}$} & \multicolumn{2}{|c|}{ Regression coefficients B B vs Expected. B* } & \multirow{2}{*}{$\frac{P \text {-value }}{* * *}$} \\
\hline & & 0.42 & 1.52 & \\
\hline & Smoking & -1.25 & 0.28 & $<0.0001$ \\
\hline & Work & 0.11 & 1.12 & 0.50 \\
\hline & Marital status & -1.34 & 0.26 & $<0.0001$ \\
\hline & GG & 0.50 & 1.65 & 0.59 \\
\hline & $A G$ & -0.05 & 0.95 & 0.80 \\
\hline & AA & reference & & \\
\hline \multirow[t]{7}{*}{ rs1042114 } & Age & 0.30 & 1.36 & 0.01 \\
\hline & Smoking & -1.28 & 0.27 & $<0.0001$ \\
\hline & Work & 0.17 & 1.19 & 0.29 \\
\hline & Marital status & -0.96 & 0.38 & $<0.0001$ \\
\hline & GG & -0.27 & 0.75 & 0.72 \\
\hline & TG & 0.01 & 1.01 & 0.95 \\
\hline & $\pi$ & reference & & \\
\hline \multirow[t]{7}{*}{ rs2236857 } & Age & 0.30 & 1.35 & 0.01 \\
\hline & Smoking & -1.25 & 0.28 & $<0.0001$ \\
\hline & Work & 0.16 & 1.18 & 0.32 \\
\hline & Marital status & -0.95 & 0.38 & $<0.0001$ \\
\hline & $\mathrm{CC}$ & -0.22 & 0.80 & 0.80 \\
\hline & $\mathrm{TC}$ & -0.25 & 0.77 & 0.77 \\
\hline & $\pi$ & reference & & \\
\hline \multirow[t]{7}{*}{ rs2236855 } & Age & 0.31 & 1.36 & 0.01 \\
\hline & Smoking & -1.24 & 0.288 & $<0.0001$ \\
\hline & Work & 0.17 & 1.19 & 0.29 \\
\hline & Marital status & -0.96 & 0.38 & $<0.0001$ \\
\hline & CC & 0.27 & 1.31 & 1.31 \\
\hline & CA & -0.02 & 0.97 & 0.97 \\
\hline & $\mathrm{AA}$ & reference & & \\
\hline \multirow[t]{7}{*}{ rs2298896 } & Age & 0.30 & 1.35 & 0.01 \\
\hline & Smoking & -1.26 & 0.28 & $<0.0001$ \\
\hline & Work & 0.18 & 1.20 & 0.27 \\
\hline & Marital status & -0.95 & 0.38 & $<0.0001$ \\
\hline & GG & -0.33 & 0.71 & 0.08 \\
\hline & $\mathrm{TG}$ & -0.26 & 0.77 & 0.09 \\
\hline & $\pi$ & reference & & \\
\hline \multirow[t]{7}{*}{ rs421300 } & Age & 0.31 & 1.37 & 0.004 \\
\hline & Smoking & -1.24 & 0.28 & $<0.0001$ \\
\hline & Work & 0.19 & 1.20 & 0.26 \\
\hline & Marital status & -0.97 & 0.37 & $<0.0001$ \\
\hline & GG & -0.25 & 0.77 & 0.19 \\
\hline & GA & -0.10 & 0.90 & 0.51 \\
\hline & $\mathrm{AA}$ & reference & & \\
\hline \multirow[t]{2}{*}{ rs529520 } & Age & 0.31 & 1.37 & 0.004 \\
\hline & Smoking & -1.23 & 0.29 & $<0.0001$ \\
\hline
\end{tabular}


Table 5 Regression analysis of the association between drug addiction features and candidate SNPs of OPRD1 gene (Continued)

\begin{tabular}{|c|c|c|c|c|}
\hline \multirow[t]{2}{*}{$\underline{\text { SNP ID }}$} & \multirow{2}{*}{$\begin{array}{l}\text { Covariate } \\
\text { Work }\end{array}$} & \multicolumn{2}{|c|}{ Regression coefficients B B vs Expected. B* } & \multirow{2}{*}{$\frac{P \text {-value }}{* * *}$} \\
\hline & & 0.18 & 1.20 & \\
\hline & Marital status & -0.94 & 0.38 & $<0.0001$ \\
\hline & $\mathrm{CC}$ & 0.23 & 1.27 & 0.20 \\
\hline & CA & 0.15 & 1.16 & 0.32 \\
\hline & AA & reference & & \\
\hline \multirow[t]{7}{*}{ rs12749204 } & Age & 0.29 & 1.34 & 0.01 \\
\hline & Smoking & -1.25 & 0.28 & $<0.0001$ \\
\hline & Work & 0.17 & 1.19 & 0.28 \\
\hline & Marital status & -0.96 & 0.38 & $<0.0001$ \\
\hline & GG & -0.25 & 0.77 & 0.37 \\
\hline & $A G$ & -0.11 & 0.89 & 0.42 \\
\hline & AA & reference & & \\
\hline \multirow[t]{7}{*}{ rs2234918 } & Age & 0.31 & 1.36 & 0.004 \\
\hline & Smoking & -1.27 & 0.28 & $<0.0001$ \\
\hline & Work & 0.19 & 1.21 & 0.25 \\
\hline & Marital status & -0.97 & 0.37 & $<0.0001$ \\
\hline & CC & -0.13 & 0.87 & 0.50 \\
\hline & $C T$ & -0.18 & 0.83 & 0.30 \\
\hline & $\pi$ & reference & & \\
\hline
\end{tabular}

* Beta coefficients

** $P$-Value $<0.05$ is considered as significant

On the other hand, our regression analysis declared that the relationship between the age of addicts, smoking and marital status with genetic variants within OPRM1, OPRD1 and OPRK1 genes may be implicated in drug addiction risk. Remarkably, it has been reported that suicide attempts can be induced by drug addiction disorder. In regard, no correlation between significant OPRM1 SNPs (rs1799971, rs609148 and rs648893) and suicide attempts behavior among 426 EuropeanAmericans addicts was found [36]. However, the real relation between genetic factors and clinical features of drug addiction is not fully elucidated, more studies and analyses are needed to understand the biological etiology of the disorder.

\section{Conclusion}

In conclusion, we propose that rs1799971 of the OPRM1 gene is a genetic risk factor for drug addiction among Jordanian males. To avoid genotyping bias in this study, we excluded the genetic association outcome caused by population stratification. This study was the first to investigate a genetic association of opioid gene variations with drug addiction in males of Arab descent. These findings may provide crucial knowledge to understand the drug addiction mechanisms in Middle Eastern population of Arab descent. Overall, the current study has examined three genes of opioid receptors that appear to be involved in the vulnerability to and the treatment of drug addiction in a Middle Eastern population of Arab descent. Finally, the results of this study provided additional clinical, epidemiological and genetic knowledge that may be useful in the context of further genetic and pharmacogenetic analyses to reduce the severity of drug consumption and improve drug abstinence.

\section{Supplementary Information}

The online version contains supplementary material available at https://doi. org/10.1186/s12888-020-03006-z.

Additional file 1: Table S1. Different genetic models analysis between candidate gene SNPS and drug addiction. Tables2. Association between drug addiction and different haplotypes

\section{Abbreviations}

OPR: Opioid receptors; HWE: Hardy-Weinberg equilibrium; DSM: Manual of Mental Disorders; DRC-PSD: Drug Rehabilitation Centre of the Jordanian Public Security Directorate; NCRA: National Centre for Rehabilitation of Addicts

\section{Acknowledgements}

We gratefully acknowledge the contribution of participating patients whose cooperation made this study possible. The authors also would like to express their gratitude to Jordan University and Science and Technology (JUST, Irbid, Jordan) for providing administrative and technical support.

\section{Authors' contributions}

L.N.A-E designed and supervised the study. L.N.A-E was responsible for data curation. L.N.A-E., D.M.R., and M.A.A., lead the implementation of the method 
and performed the data analysis. L.N.A-E., D.M.R., and M.A.A., helped with the interpretation, description of the results and drafted the manuscript. All authors read and approved the final manuscript.

\section{Funding}

The Scientific Research Support Fund (SRSF) at Jordanian Ministry of Higher Education supported this work under grant number MPH/1/43/2017.

\section{Availability of data and materials}

The complete processed SNP genotypic data for the three OPR genes is available as a supplementary file.

(https://mega.nz/file/YMBnxIAK\#3AiyPeLMzPhbsGHrdZnMUQld-y6sHOSx7OXzkjHYkE).

\section{Ethics approval and consent to participate}

This study was approved by the Human Ethics Committee of the Jordanian Ministry of Health and the Institutional Review Board (IRB) at Jordan University of Science and Technology. Written informed consent has been obtained from all participants. All methods of the current study were performed in accordance with the IRB guidelines and regulations.

\section{Consent for publication}

Not applicable.

\section{Competing interests}

The authors declare no conflict of interest.

\section{Author details}

'Department of Applied Biological Sciences, Jordan University of Science and Technology, Irbid 22110, Jordan. ${ }^{2}$ Department of Biotechnology and Genetic Engineering, Jordan University of Science and Technology, Irbid 22110, Jordan. ${ }^{3}$ Department of Anatomy, College of Medicine, King Khalid University, Abha 61421, Saudi Arabia. ${ }^{4}$ Genomics and Personalized Medicine Unit, College of Medicine, King Khalid University, Abha 61421, Saudi Arabia.

\section{Received: 21 September 2020 Accepted: 3 December 2020}

\section{Published online: 05 January 2021}

\section{References}

1. Ahmed M, UIHaq I, Faisal M, Waseem D, Taqi MM. Implication of OPRM1 A118G polymorphism in opioids addicts in Pakistan: in vitro and Insilico analysis. J Mol Neurosci. 2018;65:472.

2. Jadidi N, Nakhaee N. Etiology of drug abuse: a narrative analysis. J Addict. 2014;2014:352835.

3. Al-Eitan LN, Jaradat SA, Hulse GK, Tay GK. Custom genotyping for substance addiction susceptibility genes in Jordanians of Arab descent. BMC Rese Notes. 2012:5:497.

4. AL-Eitan L, Tarkhan A. Practical challenges and translational issues in pharmacogenomics and personalized medicine from 2010 onwards. Curr Pharmacogenom Personalized Med. 2016;14:7-17.

5. Al-Eitan LN, Jaradt SA, Hulse GK, Tay GK. Pharmacogenetic approach to treating drug dependence: serotonin transporter gene (SLC6A4) promoter polymorphisms as treatment predictors in Jordanian Arabs. Curr Pharmacogenomics Pers Med. 2012;10:293-305.

6. Bevilacqua L, Goldman D. Genes and addictions. Clin Pharmacol Ther. 2009; 85:359-61.

7. Wang SC, Chen YC, Lee CH, Cheng CM. Opioid addiction, genetic susceptibility, and medical treatments: A review. Int J Mol Sci. 2019;20:4294.

8. Ninković J, Roy S. Role of the mu-opioid receptor in opioid modulation of immune function. Amino Acids. 2013:45:9-24.

9. Randesi M, Rotrosen J, Nunes EV, Lee JD, Novo P, Levran O, et al. Variants of opioid genes and response to treatment of opioid use disorder with buprenorphine-naloxone versus extended-release naltrexone in Caucasians. Am J Drug Alcohol Abuse. 2020:1-8.

10. Colloca L, Wang Y, Martinez PE, Chang Y-PC, Ryan KA, Hodgkinson C, et al. OPRM1 rs1799971, COMT rs4680, and FAAH rs324420 genes interact with placebo procedures to induce hypoalgesia. Pain. 2019;160:1824-34.

11. Crowley JJ, Oslin DW, Patkar AA, Gottheil E, DeMariaJr PA, O'Brien CP, et al. A genetic association study of the mu opioid receptor and severe opioid dependence. Psychiatr Genet. 2003;13:169-73.
12. Mistry CJ, Bawor M, Desai D, Marsh DC, Samaan Z. Genetics of opioid dependence: A review of the genetic contribution to opioid dependence. Curr Psychiatry Rev. 2014;10:156-67.

13. Gelernter J, Kranzler H, Cubells J. Genetics of two $\mu$ opioid receptor gene (OPRM1) exon 1 polymorphisms: population studies, and allele frequencies in alcohol-and drug-dependent subjects. Mol Psychiatry. 1999;4:476-83.

14. Yuferov V, Fussell D, LaForge KS, Nielsen DA, Gordon D, Ho A, et al. Redefinition of the human kappa opioid receptor gene (OPRK1) structure and association of haplotypes with opiate addiction. Pharmacogenetics. 2004;14:793-804.

15. Crist RC, Ambrose-Lanci LM, Vaswani M, Clarke TK, Zeng A, Yuan C, et al. Case-control association analysis of polymorphisms in the $\delta$-opioid receptor, OPRD1, with cocaine and opioid addicted populations. Drug Alcohol Depend. 2013;127:122-8.

16. Mayer P, Rochlitz H, Rauch E, Rommelspacher H, Hasse HE, Schmidt S, et al. Association between a delta opioid receptor gene polymorphism and heroin dependence in man. Neuroreport. 1997;8:2547-50.

17. Nelson EC, Lynskey MT, Heat AC, Wray N, Agrawal A, Shand FL, et al. Association of OPRD1 polymorphisms with heroin dependence in a large case-control series. Addict Biol. 2014;19:111-21.

18. Xu K, Seo D, Hodgkinson C, Hu Y, Goldman D, Sinha R. A variant on the kappa opioid receptor gene (OPRK1) is associated with stress response and related drug craving, limbic brain activation and cocaine relapse risk. Transl Psychiatry. 2013;3:292.

19. Corley RP, Zeiger JS, Crowley T, Ehringer MA, Hewitt JK, Hopfer CJ, et al. Association of candidate genes with antisocial drug dependence in adolescents. Drug Alcohol Depend. 2008;96:90-8.

20. Zhang H, Kranzler HR, Yang BZ, Luo X, Gelernter J. The OPRD1 and OPRK1 loci in alcohol or drug dependence: OPRD1 variation modulates substance dependence risk. Mol Psychiatry. 2008;13:531-43.

21. American Psychiatric Association, DSM-5 Task Force. Diagnostic and statistical manual of mental disorders: DSM-5 ${ }^{\text {TM }}$ (5th ed.). American Psychiatric Publishing, Inc; 2013.

22. Nyholt DR. A simple correction for multiple testing for single-nucleotide polymorphisms in linkage disequilibrium with each other. Am J Hum Genet. 2004; $74: 765-9$

23. Li J, Ji L. Adjusting multiple testing in multilocus analyses using the eigenvalues of a correlation matrix. Heredity. 2005;95:221-7.

24. Agrawal A, Lynskey MT. Are there genetic influences on addiction: evidence from family, adoption and twin studies. Addiction. 2008;103:1069-81.

25. Wang JC, Kapoor M, Goate AM. The genetics of substance dependence. Annu Rev Genomics Hum Genet. 2012;13:241-61.

26. Al-Eitan L, Haddad Y. Emergence of pharmacogenomics in academic medicine and public health in Jordan: history, present state and prospects. Curr Pharmacogenomics Pers Med. 2014;12:167-75.

27. Alblooshi H, Hulse G, Osman W, El Kashefm A, Shawky M, Ghaferi H, et al. The frequency of DRD2 rs1076560 and OPRM1 rs1799971 in substance use disorder patients from the United Arab Emirates. Ann General Psychiatry. 2018;17:22.

28. Al-Eitan LN, Alshudaifat KM, Anani JY. Association of the DRD4 exon III and 5-HTTLPR VNTR polymorphisms with substance abuse in Jordanian Arab population. Gene. 2020;733:144267.

29. Al-Eitan LN, Jaradat SA, Qin W, Wildenauer DMB, Wildenauer DD, Hulse GK, et al. Characterization of serotonin transporter gene (SLC6A4) polymorphisms and its association with drug dependence in a Jordanian Arab population. Toxicol Ind Health. 2014;30:598-610.

30. Al-Eitan LN, Jaradat SA, Su SY, Tay GK, Hulse GK. Mu opioid receptor (OPRM1) as a predictor of treatment outcome in opiate-dependent individuals of Arab descent. Pharmacogenomics Pers Med. 2012;5:99-111.

31. Chou WY, Wang CH, Liu PH, Liu CC, Tseng CC, Jawan B. Human opioid receptor $\mathrm{A} 118 \mathrm{G}$ polymorphism affects intravenous patient-controlled analgesia morphine consumption after total abdominal hysterectomy. Anesthesiology. 2006;105:334-7.

32. Oertel BG, Kettner M, Scholich K, Renné C, Roskam B, Geisslinger G, et al. A common human $\mu$-opioid receptor genetic variant diminishes the receptor signaling efficacy in brain regions processing the sensory information of pain. J BiolChem. 2009;284:6530-5.

33. Zhang D, Shao C, Shao M, Yan P, Wang Y, Liu Y, et al. Effect of mu-opioid receptor gene polymorphisms on heroin-induced subjective responses in a Chinese population. Biol Psychiatry. 2007:61:1244-51.

34. Rezaeifar A, Dahmardeh F. The effect of OPRM1 rs648893 gene polymorphism on opioid addiction in an Iranian population in Zabol: A case-control study. Int J Basic Sci Med. 2019;4:143-7. 
35. Xuei X, Flury-Wetherill L, Bierut L, Dick D, Jr JN, Foroud T, et al. The opioid system in alcohol and drug dependence: family-based association study. Am J Med Genet B Neuropsychiatr Genet. 2007;144:877-.

36. Arias AJ, Chan G, Gelernter J, Farrer L, Kranzler HR. Variation in OPRM1 and risk of suicidal behavior in drug-dependent individuals. Am J Addict. 2012; 21:5-10.

\section{Publisher's Note}

Springer Nature remains neutral with regard to jurisdictional claims in published maps and institutional affiliations.

Ready to submit your research? Choose BMC and benefit from:

- fast, convenient online submission

- thorough peer review by experienced researchers in your field

- rapid publication on acceptance

- support for research data, including large and complex data types

- gold Open Access which fosters wider collaboration and increased citations

- maximum visibility for your research: over $100 \mathrm{M}$ website views per year

At BMC, research is always in progress.

Learn more biomedcentral.com/submissions 